\title{
Treating fracture of the clavicle
}

\author{
Patrick D.G. Henry MD
}

See also www.cmajopen.ca/content/3/4/E396

$\mathrm{H}$ istorically, one of the few golden rules of orthopedics was that midshaft clavicle fractures did not need surgical repair. Literature published predominantly within the last 10 years has challenged this accepted wisdom, and the pendulum has swung toward surgical treatment. With any major shift in medical practice, it is appropriate to continue to evaluate the practice. A rigorous systematic review and meta-analysis recently published in CMAJ Open found that rates of complications of surgery and unplanned secondary operations were high, regardless of the treatment approach used. ${ }^{1}$ It would seem, on the surface, that current evidence does not support routine fixation for displaced midshaft clavicle fractures.

A closer look at the linked study reveals that all of the included trials that compared operative with nonoperative treatment had an uncertain to high risk of bias and were of low overall quality. Although complication and reoperation rates were similar between surgical and nonsurgical groups, the type of complication and reasons for reoperation were different, something that is equally - and perhaps more - relevant than merely the number of complications. The authors found that the most common adverse effect of surgical management was the development of irritation from metal fixation hardware, necessitating removal, whereas the most common cause of secondary operation after nonoperative treatment was symptomatic nonunion. Symptomatic hardware is typically a nuisance to patients, whereas symptomatic nonunion has been shown to be debilitating. ${ }^{2}$ Furthermore, the surgical solution to irritating hardware is simple; the solution to nonunion is frequently far more difficult than surgery for the acute fracture, and with results that have been previously shown to be inferior to acute fixation. ${ }^{3}$

Another important problem that is reduced by surgery is the incidence of malunion. Sequelae such as shortening of the clavicle and rotational deformities cause changes in scapular positioning. This affects the integrity of both the sternoclavicular and acromioclavicular articulations, which can lead to weakness, pain, a sensation of abnormal posturing and poor functionality of the arm, shoulder and hand. ${ }^{4-6}$ The median follow-up period of the studies in the linked meta-analysis was one year; however, the symptoms of malunion are often subtle and may not be noticeable within one year unless explicitly looked for or until patients attempt to return to sport or other physical activity. ${ }^{7}$ They are also permanent. Furthermore, the surgical management of malunion is, as with nonunion, typically a more challenging operation than acute open reduction and internal fixation; managing malunion requires osteotomy and reshaping of the clavicle, followed by open reduction and internal fixation.

The review found functional Disabilities of the Arm, Shoulder and Hand (DASH) Outcome Measure scores to be superior at one year in the surgical group, which was statistically significant but did not meet the threshold for a minimum clinically important difference. ${ }^{1}$ Although the DASH Outcome Measure was the most common measurement tool used in the included studies, it has not been validated for clavicle fractures and has been shown to have overall good but not excellent responsiveness. ${ }^{8}$ It is possible that small changes in DASH scores could translate into much larger changes if a more responsive tool were used to measure functional outcomes. Although this is conjecture, bias in the included studies was high enough that functional outcomes were not judged as being essentially "the same" (from the patient's viewpoint)

\section{KEY POINTS}

- Most midshaft fractures of the clavicle can be treated nonoperatively. However, evidence suggests that a subset of these fractures benefits from primary operative fixation.

- Rates of complications and secondary operations are high, regardless of treatment approach.

- More well-conducted research is needed to better define clear indications for surgical management.

- For now, ensuring that the patient is well-informed of the known risks and benefits will lead to individualized treatment strategies. 
with or without surgery, as the results of the linked study would seem to indicate.

Although the focus of the linked study was on complications and reoperations, the article highlighted indications for surgery as an important issue. It shows that the absolute indications for surgical intervention for midshaft fracture of the clavicle are still unknown. It is clear that most patients with midshaft clavicle fractures treated conservatively will heal and "do well"; however, some do not. Although it seems that displacement alone is not a strong enough predictor of a poor outcome for it to be considered a clear risk factor, it is one of the important factors. A key inference from the linked study is that there is still more work to do in the search for the other patient characteristics (and combinations of those characteristics) that should lead us to recommend primary operative fixation for midshaft fracture of the clavicle.

Although diminishing funding opportunities are the largest barrier to producing a high-quality study, surgical trials also have high costs (particularly those involving patients with trauma) and problems inherent to blinding. Despite these real challenges (also including difficulties in recruitment, ${ }^{9}$ lack of surgeons able or willing to participate, variable surgical skill levels and techniques, and patient crossovers and/or loss to follow-up), the call for higher quality studies in orthopedics has been heard for some time ${ }^{10}$ and cannot be ignored. Designing and conducting better studies is the only way that areas of controversy, such as those outlined in this article, can be reconciled.

I agree with the authors of the linked study that the routine performance of surgery for displaced midshaft clavicles should not be recommended; however, I would add that the avoidance of surgery for all displaced midshaft clavicle fractures should also not be routine practice. For now, because research has not yet revealed clear indications for surgical intervention, the answer is to ensure that the patient is well-informed about current evidence through a discussion of risks and benefits that can help guide an individualized treatment plan. The choice will be different for patients with similar imaging. Some patients may want to avoid the chance of two surgeries, some will want to ensure their clavicle is anatomically aligned and some simply may not want a scar.

\section{References}

1. Devji T, Kleinlugtenbelt Y, Evanview N, et al. Operative versus nonoperative interventions for common fractures of the clavicle: a meta-analysis of randomized controlled trials. CMAJ Open 2015;3:E396-405.

2. Der Tavitian J, Davison JNS, Dias J. Clavicular fracture nonunion surgical outcome and complications. Injury. Injury 2002; 33:135-43.

3. Potter JM, Jones C, Wild LM, et al. Does delay matter? The restoration of objectively measured shoulder strength and patient-oriented outcome after immediate fixation versus delayed reconstruction of displaced midshaft fractures of the clavicle. J Shoulder Elbow Surg 2007;16:514-8.

4. Lazarides S, Zafiropoulos G. Conservative treatment of fractures at the middle third of the clavicle: the relevance of shortening and clinical outcome. J Shoulder Elbow Surg 2006;15:191-4.

5. Ledger M, Leeks N, Ackland T, et al. Short malunions of the clavicle: an anatomic and functional study. J Shoulder Elbow Surg 2005; 14:349-54.

6. McKee MD, Pedersen EM, Jones C, et al. Deficits following non-operative treatment of displaced, midshaft clavicle fractures. J Bone Joint Surg Am 2006;88:35-40.

7. McKee MD, Wild LM, Schemitsch EH. Midshaft malunions of the clavicle. J Bone Joint Surg Am 2003;85-A:790-7.

8. Beaton DE, Katz JN, Fossel AH, et al. Measuring the whole or the parts? Validity, reliability, and responsiveness of the Disabilities of the Arm, Shoulder and Hand outcome measure in different regions of the upper extremity. J Hand Ther 2001;14:128-46.

9. Cook JA, Ramsay CR, Norrie J. Recruitment to publicly funded trials - Are surgical trials really different? Contemp Clin Trials 2008;29:631-4

10. Obremskey WT, Pappas N, Attallah-Wasif E, et al. Level of evidence in orthopaedic journals. J Bone Joint Surg Am 2005;87: 2632-8.

Affiliation: Division of Orthopaedic Surgery, Department of Surgery, Sunnybrook Health Sciences Centre, University of Toronto, Toronto, Ont. 